Also available at http://amc.imfm.si

ISSN 1855-3966 (printed edn.), ISSN 1855-3974 (electronic edn.)

ARS MATHEMATICA CONTEMPORANEA 5 (2012) 99-105

\title{
HL-index of a graph
}

\author{
Gašper Jaklič \\ FMF and IMFM, University of Ljubljana, and PINT, University of Primorska \\ Jadranska 19, 1000 Ljubljana, Slovenia \\ Patrick W. Fowler \\ Department of Chemistry, University of Sheffield, Sheffield S3 7HF, UK \\ Tomaž Pisanski \\ FMF and IMFM, University of Ljubljana, and PINT, University of Primorska \\ Jadranska 19, 1000 Ljubljana, Slovenia
}

Received 15 November 2010, accepted 26 April 2011, published online 12 December 2011

\begin{abstract}
Let $G$ be a simple, connected graph with $n$ vertices and eigenvalues $\lambda_{1}>\lambda_{2} \geq \ldots \geq$ $\lambda_{n}$. If $n$ is even, define $H=n / 2$ and $L=H+1$. If $n$ is odd, define $H=L=(n+1) / 2$. Define the HL-index of $G$ to be $R(G)=\max \left(\left|\lambda_{H}\right|,\left|\lambda_{L}\right|\right)$. The eigenvalues $\lambda_{H}$ and $\lambda_{L}$ appear in chemical graph theory in the study of molecular stability. In this paper, bounds on HL-index for chemical and general graphs are studied. It is shown that there exist graphs with arbitrarily large HL-index.
\end{abstract}

Keywords: HL-index, graph spectrum, HOMO-LUMO map.

Math. Subj. Class.: 15A18, 05C50, 05C90

\section{Introduction}

First, recall the definition of the graph spectrum. Let $G$ be a simple, connected graph on $n$ vertices and $A(G)$ its adjacency matrix. The collection of eigenvalues of $A(G)$ is called the spectrum, $\operatorname{spec}(G)$, of $G$. Since $A(G)$ is a symmetric matrix, its spectrum is real and can be described as follows: $\operatorname{spec}(G)=\left\{\lambda_{1}, \lambda_{2}, \ldots, \lambda_{n}\right\}$, where $\lambda_{1}>\lambda_{2} \geq \ldots \geq \lambda_{n}$. Since $A(G)$ has a zero diagonal, $\sum_{i} \lambda_{i}=0$. To indicate eigenvalue multiplicities, $\operatorname{spec}(G)$ can be rewritten as

$$
\operatorname{spec}(G)=\left\{\left[\mu_{1}\right]^{a_{1}},\left[\mu_{2}\right]^{a_{2}}, \ldots,\left[\mu_{s}\right]^{a_{s}}\right\},
$$

E-mail addresses: gasper.jaklic@fmf.uni-lj.si (Gašper Jaklič), P.W.Fowler@sheffield.ac.uk (Patrick W. Fowler), tomaz.pisanski@fmf.uni-lj.si (Tomaž Pisanski) 
where $\mu_{1}>\mu_{2}>\ldots>\mu_{s}$ are distinct eigenvalues of $A(G)$ and $a_{1}, a_{2}, \ldots, a_{s}$ are their multiplicities. Note that $a_{1}=1$ for connected graphs, since they have a simple principal eigenvalue.

In linear algebra and in spectral graph theory, particular attention is paid to the principal (Perron) eigenvalue $\lambda_{1}$. In graph theory, the minimal eigenvalue $\lambda_{n}$ and the second eigenvalue, $\lambda_{2}$, have also been studied. Apart from classical bounds, not much is known in general about the remaining eigenvalues, although there has been a great deal of work on spectra of specific classes of graphs of importance in chemistry and elsewhere [4, 10, 5, 3].

In theoretical chemistry the middle eigenvalues play an important role in the Hückel model of $\pi$-electron systems. For even $n$ we define $H:=n / 2$ and $L:=H+1$. If $n$ is odd, we define $H:=L:=(n+1) / 2$. In chemical graph theory, the eigenvalue difference $\lambda_{H}-\lambda_{L}$ is called the HOMO-LUMO gap of $G$ and is related to the kinetic stability of a molecule. HOMO and LUMO denote Highest Occupied Molecular Orbital and Lowest Unoccupied Molecular Orbital, respectively. There is a direct correspondence between eigenvalues and the corresponding eigenvectors of the molecular graph and the orbital energies and molecular orbitals in the Hückel model.

Given the set of eigenvalues, the ground-state electron configuration of the molecule and hence of the graph is determined by application of three rules: the Aufbau Principle (fill orbitals in order of decreasing eigenvalue), the Pauli Principle (no orbital may contain more than two electrons), and Hund's Rule of Maximum Multiplicity (no orbital receives a second electron before all orbitals degenerate with it have each received one). A molecule and its graph are open shell if any orbitals/eigenvectors are partially occupied, and closed shell otherwise. For neutral molecules, the order of the molecular graph, $n$, is equal to the number of electrons in the $\pi$ system. The (fully or partly) occupied orbital of highest energy (corresponding to eigenvalue $\lambda_{H}$ ) is the HOMO. The (partly or fully) occupied orbital of lowest energy (corresponding to eigenvalue $\lambda_{L}$ ) is the LUMO. If there are partially occupied orbitals, HOMO and LUMO coincide; a partially occupied orbital is also known as a SOMO (singly occupied molecular orbital).

The pair of HOMO-LUMO eigenvalues $\left(\lambda_{H}, \lambda_{L}\right)$ may be represented as a point in a plane. Thus, for a family of graphs $\mathcal{G}$ we get a set of points. Such a diagram in the HOMO-LUMO plane is called the HOMO-LUMO map of $\mathcal{G}$ (see [7, 8]). Furthermore, the right triangle $T_{C}$ with vertices $(-1,-1),(1,-1),(1,1)$ is called the chemical triangle. A connected graph with maximum valence at most 3 is a chemical graph. Experimental evidence shows that most chemical graphs are mapped into the chemical triangle [7]. For instance, in [8] the authors verify this for many fullerenes ([9]), and in particular, it can be proved to be true for chemical trees, i.e., trees with maximal valence $\leq 3$. However, it is not true that all chemical graphs possess this property. The smallest counterexample is the Heawood graph, a well-known 3-regular graph that is the Levi graph of the Fano plane, the 6-cage, and the skeleton of the smallest "polyhedral" polyhex torus. Its spectrum is: $\left\{[3]^{1},[\sqrt{2}]^{6},[-\sqrt{2}]^{6},[-3]^{1}\right\}$ (see, e.g., $[1,2]$ ). Hence the Heawood graph falls outside the chemical triangle $T_{C}$. So far, this is the only chemical graph that we know to fall outside $T_{C}$. Exhaustive search reveals no other example with 19 or fewer vertices, and no other exception amongst 3 -regular graphs with 24 or fewer vertices. Once the degree constraint is relaxed, graphs $G$ with $R(G)>1$ appear in large numbers ( 2 for $n=6,119$ for $n=8$, 37 for $n=9,151062$ for $n=10$, see [7]).

The HOMO-LUMO radius or HL-index ([7]) is defined as

$$
R(G):=\max \left\{\left|\lambda_{H}\right|,\left|\lambda_{L}\right|\right\} .
$$


The theorem about chemical trees can be restated as follows.

Theorem 1.1 ([7]). For each chemical tree T, the HL-index is bounded by 1 ,

$$
R(T) \leq 1
$$

For general graphs, no such bound is known. By the Gerschgorin and Cauchy interlacing theorems one can easily prove that the HL-index of a graph is bounded by its maximal degree or the average valence [4]. For bipartite and pseudo-bipartite graphs, tighter bounds were obtained in [7]. (A pseudo-bipartite graph is not bipartite, but like a bipartite graph it has $\lambda_{H}=-\lambda_{L}$.)

Theorem 1.2 ([7]). Let $G$ be a bipartite or pseudo-bipartite graph with $n$ vertices and $m$ edges, and let $\bar{d}:=2 m / n$ denote its average degree. Then

$$
0 \leq R(G) \leq \sqrt{\bar{d}}
$$

Thus, in particular, if $G$ is a chemical bipartite or pseudo-bipartite graph,

$$
0 \leq R(G) \leq \sqrt{3}
$$

Now let $\mathcal{G}$ be a family of graphs. Define the HL-index of $\mathcal{G}, R(\mathcal{G})$, to be the maximum value of $R(G)$ for $G \in \mathcal{G}$.

Theorem 1.3. Let $\mathcal{G}_{D}$ be the family of graphs with maximal degree at most $D$. Then

$$
0 \leq R\left(\mathcal{G}_{D}\right) \leq D
$$

The equality in the lower bound is reached for many graphs, whilst the equality in the upper bound is reached only for the complete graph $K_{2}$.

Proof. The lower bound is obvious from the non-negativity of the HL-index. The equality is reached for the complete bipartite graph $K_{2,2}$, amongst many others. The Gerschgorin theorem yields the upper bound. Here, equality is obviously reached for the complete graph on 2 vertices $K_{2}$. There are no other such graphs. In such a case, the absolute value of the "middle" eigenvalue $\lambda_{H}$ or $\lambda_{L}$ should be $D$. But since $\left|\lambda_{i}\right| \leq D$ for all $i$, at least half of the eigenvalues should be equal to $D$ or $-D$. Since by the Perron-Frobenius theorem for simple connected graphs the Perron eigenvalue is simple, this is possible only for $n=2$ and the graph $K_{2}$.

Since for the Heawood graph $G, R(G)=\sqrt{2}$, the upper bound is in general at least $\sqrt{2}$ and less than 3 for chemical graphs.

Theorem 1.4. Let $\mathcal{C}=\mathcal{G}_{3}$ be the family of chemical graphs. Then

$$
\sqrt{2} \leq R(\mathcal{C})<3
$$




\section{Main result}

The HL-index of a family of graphs is bounded from above by a function of its maximum degree. Here we show that the HL-index may be arbitrarily large.

Theorem 2.1. For each positive constant $K>0$ there exists a connected graph $G(K)$ such that the HL-index of $G(K)$ is greater than $K$,

$$
R(G(K))>K
$$

Proof. We will use Paley graphs and their spectral properties (see, e.g., [1, 2]). Let $q=p^{m}$ be a prime power of the form $q=4 t+1, t \in \mathbb{N}$. The graph $P(q)$ is defined on the finite field $F(q)$ with $q$ elements. Its vertex set is $F(q)$, and two vertices are adjacent if and only if their difference is a square in the field. The Paley graph $P(q)$ is self-complementary and strongly regular. The eigenvalues of the Paley graph $P(q)$ are

$$
\left\{\left[\frac{q-1}{2}\right]^{1},\left[\frac{-1+\sqrt{q}}{2}\right]^{\frac{q-1}{2}},\left[\frac{-1-\sqrt{q}}{2}\right]^{\frac{q-1}{2}}\right\}
$$

Hence $R(P(q))=\lambda_{H}=\lambda_{L}=(\sqrt{q}-1) / 2$. Take $q>(2 K+1)^{2}$, and the proof is concluded.

A Paley graph has an odd number of vertices and hence $\lambda_{H}=\lambda_{L}$ by definition. One can ask a natural question: Is it possible to find a sequence of even-order connected graphs with increasing HL-index? The answer to this question is in the affirmative.

For instance, take any connected, regular, bipartite graph $G$ that has exactly 4 distinct eigenvalues. Cvetković, Doob, and Sachs [4, p. 166] proved that $G$ has to be a Levi graph of a symmetric $2-(v, k, \lambda)$ design. Its spectrum is (see [6])

$$
\left\{[k]^{1},[\sqrt{k-\lambda}]^{v-1},[-\sqrt{k-\lambda}]^{v-1},[-k]^{1}\right\},
$$

and therefore its HL-index is equal to $\sqrt{k-\lambda}$. For $k=n+1, v=n^{2}+n+1, \lambda=1$, the designs are finite projective planes, which exist for prime powers $n=p^{m}$. Hence $\sqrt{k-\lambda}=\sqrt{n}$ is arbitrarily large.

\section{Questions}

Let us conclude the paper with some interesting questions.

1. Is the Heawood graph the only chemical graph outside the chemical triangle $T_{C}$ ?

2. Let $\mathcal{C}=\mathcal{G}_{3}$ be the family of chemical graphs. By Thm. $1.4, \sqrt{2} \leq R(\mathcal{C})<3$. How large can $R(\mathcal{C})$ be?

3. It would be interesting to determine the graphs $G$ on $n$ vertices with maximal HLindex $R(G)$. For small numbers of vertices, $n$, the graphs maximizing $R(G)$ (or if there are many, the numbers of such graphs) are listed in Table 1, and examples are shown in Figure 1.

Here $G_{6,1}$ is the pentagonal pyramid (wheel with 5 spokes), $G_{8,1}$ is the two-dimensional subdivision of the tetrahedron, and $G_{10,1}$ is the complement of the Petersen 


\begin{tabular}{c|c|c}
$n$ & $R(G)$ & graphs \\
\hline 2 & 1 & $K_{2}$ \\
\hline 3 & 1 & $K_{3}$ \\
\hline 4 & 1 & $K_{4}-e, K_{4}$ \\
\hline 5 & 1 & $G_{5,1}, G_{5,2}, G_{5,3}, G_{5,4}, G_{5,5}=K_{5}$ \\
\hline 6 & $\sqrt{6}-1$ & $G_{6,1}$ \\
\hline 7 & 1 & 109 graphs, the one with most edges being $K_{7}$ \\
\hline 8 & $\phi=(1+\sqrt{5}) / 2$ & $G_{8,1}$ \\
\hline 9 & $\sqrt{2}$ & $G_{9,1}, G_{9,2}, G_{9,3}$ \\
\hline 10 & 2 & $G_{10,1}$ \\
\hline
\end{tabular}

Table 1: Graphs on $n$ vertices with maximal HL-index.

graph. When the maximal HL-index for a given $n$ is equal to 1, the complete graph $K_{n}$ achieves that maximum (as $\operatorname{spec}\left(K_{n}\right)=\left\{[n-1]^{1},[-1]^{n-1}\right\}$ ), and clearly is the unique graph with the maximal number of edges $m$ that does so.

4. Determine the graphs $G$ with $n$ vertices and $m$ edges with maximal HL-index $R(G)$.

5. Since the number of adjacency matrices of chemical graphs is countable, the same holds for the image of the HOMO-LUMO map. Does there exist a simply connected region with positive area in the chemical triangle that contains no points $\left(\lambda_{H}, \lambda_{L}\right)$ ?

\section{Acknowledgements}

GJ and TP thank ARRS for a grant P1-294. PWF thanks the Royal Society for an award under the RS/Wolfson Research Merit Scheme. TP thanks Peter Šemrl for a fruitful discussion. 


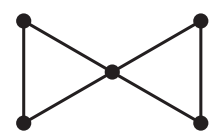

$G_{5,1}$

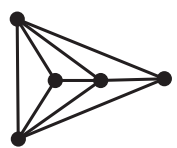

$G_{5,4}$

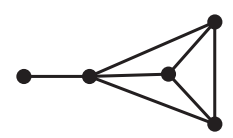

$G_{5,2}$

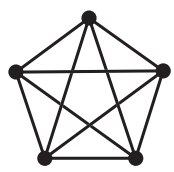

$G_{5,5}$

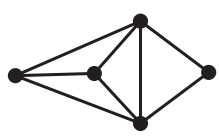

$G_{5,3}$

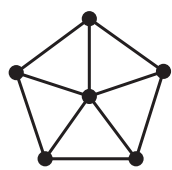

$G_{6,1}$

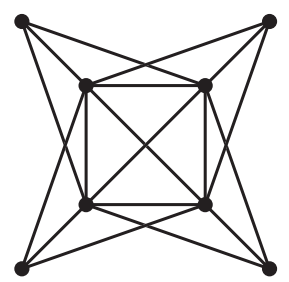

$G_{8,1}$

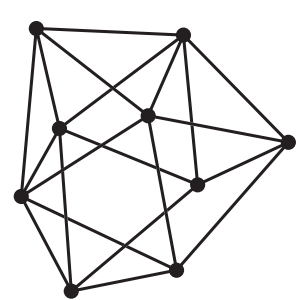

$G_{9,1}$

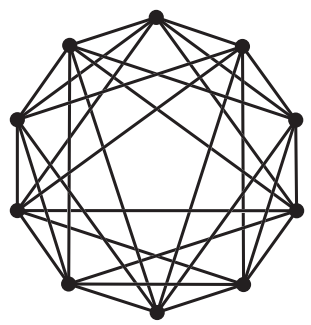

$G_{10,1}$

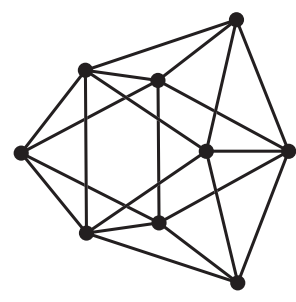

$G_{9,2}$

$G_{9,3}$

Figure 1: Graphs $G_{n, i}$ with maximal HL-index. 


\section{References}

[1] A. E. Brouwer, A. M. Cohen and A. Neumaier, Distance-regular Graphs, Ergebnisse der Mathematik 3.18, Springer, Heidelberg, 1989.

[2] A. E. Brouwer, Graph descriptions, http://www.win.tue.nl/ aeb/graphs/ index. html, (last accessed 12.11.2010).

[3] F. R. K. Chung, Spectral Graph Theory, CBMS Regional Conference Series in Mathematics, American Mathematical Society, 1997.

[4] D. M. Cvetković, M. Doob and H. Sachs, Spectra of Graphs, V.E.B. Deutscher Verlag der Wissenschaften, Berlin, 1979.

[5] D. Cvetković, P. Rowlinson and S. Simić, An Introduction to the Theory of Graph Spectra, London Mathematical Society Student Texts, Cambridge University Press, 2009.

[6] E. R. van Dam, Regular graphs with four eigenvalues, Linear Algebra Appl. 226-228 (1995) 139-162.

[7] P. W. Fowler and T. Pisanski, HOMO-LUMO maps for chemical graphs, MATCH Commun. Math. Comput. Chem. 64 (2010), 373-390.

[8] P. W. Fowler and T. Pisanski, HOMO-LUMO maps for fullerenes, Acta Chim. Slov., 57 (2010), 513-517.

[9] P. W. Fowler and T. Pisanski, Leapfrog fullerenes and Clar polyhedra, J. Chem. Soc., Faraday Trans. 90 (1994), 2865-2871.

[10] I. Gutman and O. Polansky, Mathematical Concepts in Organic Chemistry, Springer, Berlin, 1986. 